Gerión. Revista de Historia Antigua

ISSN: 0213-0181

\title{
La anaciclosis polibiana, principio de autoridad durante el periodo constituyente de los Estados Unidos
}

\author{
Clelia Martínez Maza ${ }^{1}$
}

Recibido: 6 de marzo de 2019 / Aceptado: 27 de mayo de 2019

Resumen. Uno de los rasgos esenciales de la Constitución de los Estados Unidos es la separación de poderes entre el poder ejecutivo, legislativo y judicial, solo posible gracias al sistema de pesos y contrapesos. Los padres fundadores propusieron este mecanismo y el principio del gobierno mixto como instrumentos eficaces para prevenir la amenaza de la "anaciclosis", la degeneración natural de las tres formas simples de gobierno. La fórmula, tal y como la describe Polibio en el libro VI de sus Historias, tuvo una influencia decisiva en los debates del periodo constituyente.

Palabras clave: Polibio; periodo constituyente; padres fundadores; gobierno mixto.

\section{[en] The Power of the Polybian Anacyclosis in the Constitutional Period of USA}

Abstract. One of the central features of the United States' Constitution is the separation of powers. The division of responsibilities into legislative, executive and judicial branches is only possible through a system of checks and balances. The Founding Fathers proposed this principle and a mixed government for the new republic as powerful weapons to prevent the threat of "anacyclosis", the natural degeneration of the three simple forms of government. Polybius's explanation of "anacyclosis", found in Book VI of the Histories, had a decisive influence as we can see in the Constitutional Debates.

Keywords: Polybius; Federal Period; Founding Fathers; Mixed Government.

Sumario. 1. Introducción. 2. Propuestas para una nueva república: gobierno mixto y separación de poderes. 3. El poder didáctico de la anaciclosis. 4. Polibio en los círculos intelectuales de las colonias. 5. Esparta y Roma, inspiración del futuro gobierno mixto. 6. Referencias bibliográficas.

Cómo citar: Martínez Maza, C. (2019): La anaciclosis polibiana, principio de autoridad durante el periodo constituyente de los Estados Unidos, en Gerión 37/2, 473-487.

$1 \quad$ Universidad de Málaga.

E-mail: martinezm@uma.es 


\section{Introducción}

Desde su mismo origen, una de las características fundamentales de la Constitución de los Estados Unidos es la separación de poderes, el principio de que los poderes legislativo, ejecutivo y judicial deben ser distintos y estar separados, deben trabajar con capacidad autónoma. Para lograrlo hay que habilitar instrumentos de control y equilibrio que eviten cualquier abuso de poder por parte de las restantes ramas del gobierno. Podemos considerar a James Madison, delegado por Virginia en la convención constituyente celebrada en Filadelfia entre mayo y septiembre de 1787, como uno de los principales defensores de la inclusión de este principio en el ordenamiento constitucional de la nueva república. J. Madison defendió por escrito este sistema de controles y equilibrios (checks and balances) ${ }^{2}$ en la colección de artículos de prensa conocida como El Federalista, órgano de expresión de los partidarios del federalismo centralizador. ${ }^{3}$ El número 51 de esta publicación periódica, correspondiente al 6 de febrero de 1788, lleva el descriptivo título de: "La estructura de Gobierno debe proporcionar los controles y equilibrios adecuados entre los diferentes departamentos". En su número 47, dedicado a "la estructura del Nuevo gobierno y la distribución de poderes entre sus diferentes partes", llega a declarar que ninguna verdad política posee un valor intrínseco mayor. ${ }^{4}$

Entre los especialistas en Historia Moderna se reconoce a Montesquieu como la fuente de inspiración directa de este sistema y, sobre todo, El espiritu de las leyes, publicada en 1748, la obra de referencia contemporánea más valorada por los intelectuales americanos dado que, en su libro undécimo, queda articulada con detalle esta teoría. ${ }^{5} \mathrm{~J}$. Madison, al igual que otros padres fundadores, consideraba al filósofo francés "el oráculo que siempre se consulta y se cita sobre esta materia" ${ }^{6}$ No cabe duda de que los estadistas americanos necesitaban encontrar un precedente de prestigio que legitimara su propuesta de gobierno republicano absolutamente novedosa en la praxis política contemporánea. Pero, a pesar de las elogiosas referencias de las que es objeto Montesquieu, en mi opinión, resulta discutible aceptar que fuera la fuente directa y exclusiva y que su influencia hubiera sido cualitativamente decisiva. Por ejemplo, cuando J. Madison cita a Montesquieu como el oráculo de la doctrina de la separación de poderes, admite que: "no es el autor de este precepto invaluable en la ciencia de la política, (pero) tiene el mérito de mostrarlo y recomendarlo de la manera más efectiva para la atención de la humanidad". ${ }^{7}$ Cierto es que Montesquieu fue mencionado en varias ocasiones durante los debates en la

2 Esta expresión alude en español al sistema de pesos y contrapesos o control y equilibrio de poderes y constituye uno de los elementos esenciales del ordenamiento político de los Estados Unidos, garante del principio de separación de poderes. Cada uno de los tres poderes queda investido de competencias que permiten limitar la capacidad de acción de los restantes con objeto de evitar una excesiva concentración de poder. Sobre su implantación en el gobierno de los EE.UU.: Vile 1998, 181-192. Todas las traducciones de las obras comentadas, salvo indicación expresa en nota a pie de página, son obra mía.

McLean - McLean 1788.

4 Aunque como firmante de los artículos de El Federalista aparece Publius, pseudónimo colectivo que escondería a los tres redactores de la colección, J. Jay, J. Madison y A. Hamilton, los análisis filológicos han podido identificar a Madison como autor de los números aquí mencionados. En este artículo utilizaremos el pseudónimo para mayor comodidad.

5 Las incesantes referencias a Montesquieu en la política del momento han sido recogidas y analizadas por: Cohler 1988, 75-80; Lutz 1988, 142-147; Rahe 1992, 233; Carrese 2003, 131-135; Nelson, 2004, 155-176.

Publius 1788a.

7 Publius 1788a. 
convención constituyente, pero se recurrió a su autoridad tan solo una vez para sustentar el principio de separación de poderes. ${ }^{8}$

Por las mismas fechas, en el trabajo que John Adams dedica a la defensa de las Constituciones, publicado en enero de 1787, el único pasaje que recupera a Montesquieu aparece en la carta XXVIII, que tiene como protagonista al filósofo, y tan solo para incluir a modo de cita un fragmento de El espíritu de las leyes sin comentario ni exégesis alguna. ${ }^{9}$ Este desinterés contrasta con la atención que J. Adams dedica a otros pensadores que proponen la separación de poderes antes que Montesquieu. Es el caso de J. Harrington, al que J. Adams glosa y comenta con mucho más detalle en este mismo trabajo. ${ }^{10}$ El reconocimiento alcanzado por J. Harrington en los ambientes intelectuales de las colonias vino de la mano de su obra La República de Oceana, publicada en 1656, en la que presentaba una utopía gobernada bajo la fórmula de gobierno mixto, con equilibrio entre la monarquía, la aristocracia y la democracia casi un siglo antes de El espiritu de las leyes. ${ }^{11} \mathrm{~J}$. Adams también se manifiesta deudor de la obra de J. Swift y recuerda en una de las cartas que le dedica que, aunque muy poco reconocido en el ámbito de la política, debería ser recuperado en torno a este asunto, y muy especialmente su obra Contests and Dissensions between the Nobles and Commons of Athens and Rome, publicada en 1701. En ella, J. Swift señalaba que:

Los mejores legisladores de todos los tiempos coinciden en que el poder absoluto que originariamente descansa en un solo órgano es una empresa demasiado grande como para cederla a un solo hombre o asamblea; y por lo tanto ... deberá mantenerse como regla eterna en política, que haya un equilibrio de poder mantenido cuidadosamente en el seno de cada Estado. ${ }^{12}$

J. Adams recordaba asimismo en esta carta que J. Swift citaba a Polibio cuando enunciaba que "el mejor gobierno es el que consta de tres formas, regno, optimatium et populi imperio". ${ }^{13}$

No obstante este reconocimiento mínimo -pero explícito- a los pensadores contemporáneos, los registros de la convención constituyente de Filadelfia y El Federalista demuestran la inclinación de los delegados por llevar al debate, como argumento de autoridad, a las fuentes antiguas de las que habían bebido J. Swift o el propio Montesquieu y que este último recupera para contrastar sus hipótesis. ${ }^{14}$ Entre los referentes del mundo clásico sobresale de manera incontestable el autor clásico de mayor prestigio entre los intelectuales del momento, Polibio, ${ }^{15}$ cuya fórmula del gobierno mixto aparece ahora idealizada como el arquetipo más apropiado para las necesidades del nuevo Estado. ${ }^{16}$ En efecto, se trata de uno de los conceptos de mayor y mejor acogida en el período constituyente. ${ }^{17}$ Ya desde tiempos de la Revolución se

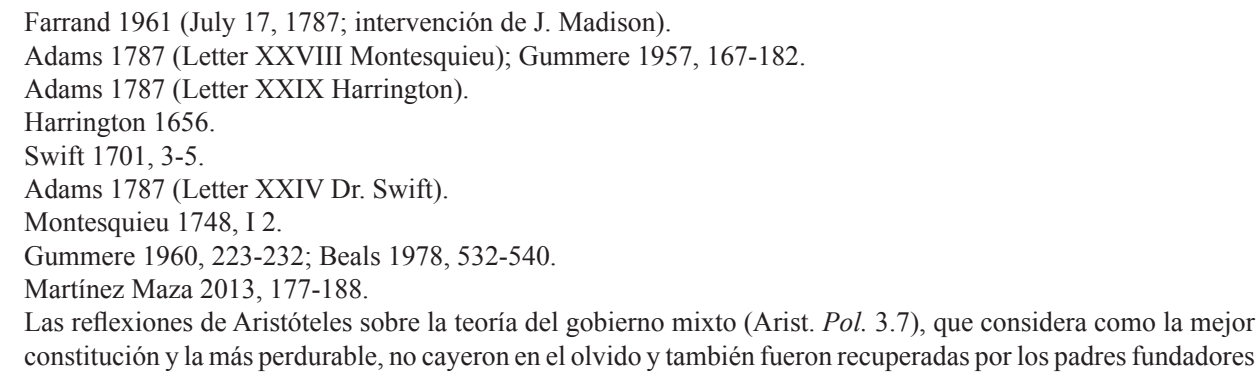


propone un gobierno mixto cuyas instituciones no fueran por completo monárquicas ni tampoco puramente democráticas. Y así, en 1772, J. Adams declaraba que el mejor gobierno del mundo era el mixto. ${ }^{18} \mathrm{Y}$ en 1776 , año de la independencia, instaba a Carolina del Norte y Virginia a establecer gobiernos mixtos en sus nuevas constituciones. ${ }^{19}$

\section{Propuestas para una nueva república: gobierno mixto y separación de poderes}

En líneas generales, los federalistas sostuvieron, por un lado, que cuanto más perfecta y ponderada fuera la combinación de fórmulas simples de gobierno (monarquía, aristocracia, democracia), mayor estabilidad lograría el Estado. ${ }^{20}$ Por otro, que el éxito de la fórmula mixta radicaba en el principio de separación de poderes, que debía quedar garantizado con el establecimiento de mecanismos de control suficientes. ${ }^{21}$ De este modo, todos y cada uno de dichos poderes quedarían protegidos de la intromisión de los restantes y de una posible concentración de competencias a favor de uno de ellos. J. Adams declaraba en un enunciado que evoca claramente a Polibio: "los hombres vigilantes y controlándose unos a otros son la única garantía, el poder debe oponerse al poder, el interés al interés". ${ }^{22} \mathrm{Y}$ en el mismo sentido se manifestaba J. Madison en un enunciado que terminó por convertirse en una de las máximas más célebres que justificaba el sistema de separación y equilibrios:

Si los hombres fueran ángeles, no sería necesario un gobierno. Si los ángeles gobernaran a los hombres, no sería necesario ningún control interno o externo sobre el gobierno. Al instituir un gobierno administrado por hombres sobre hombres, la gran dificultad es la siguiente: hacer que el gobierno sea capaz de controlar a los gobernados y al mismo tiempo obligarlo a controlarse a sí mismo. ${ }^{23}$

\section{El poder didáctico de la anaciclosis}

A juicio de los padres fundadores, que siguen fielmente el libro VI de las historias de Polibio, el gobierno mixto era la única fórmula política capaz de ofrecer una solución a la amenaza que ya había detectado el historiador griego en los gobiernos del mundo antiguo y que ahora también percibían los estadistas americanos en el escenario político en el que vivían, una amenaza que había que evitar a toda costa y

\footnotetext{
durante los debates de la convención constituyente. De hecho, Madison propuso la creación de un comité encargado de seleccionar libros de consulta en el Congreso y entre los títulos dedicados a teoría política que se proponen encontramos la Política de Aristóteles: Madison 1783.

18 Butterfield 1961, vol. II, 58.

19 Adams 1776.

20 Esta fórmula fue discutida en numerosas sesiones de la convención federal: Farrand 1961 (May 31 1787; June 4, 7, 16, 1787). También fue discutida en distintas convenciones estatales. Véase la convención de Massachusetts: Elliot 1968, II (January 22, 1788); en la convención de Virginia: Elliot 1968, III (June 4, 1788).

21 Martínez Maza 2013, 200-203.

22 Adams 1787 (Letter XLIX Crotona); del mismo modo, Polibio (Plb. 6.5) atribuye la estabilidad del gobierno de Licurgo al contrapeso y al control ejercido por cada uno de los órganos de gobierno de Esparta.

23. Publius $1788 \mathrm{~b}$.
} 
que modeló ese sistema de equilibrio y separación de poderes: el imparable recorrido cíclico de la anaciclosis, un ciclo en el que se distinguían las tres fases de politeia a las que se veía abocada una constitución de manera natural: el crecimiento ( $\alpha$ ǔ $\xi \varepsilon \sigma ı)$,

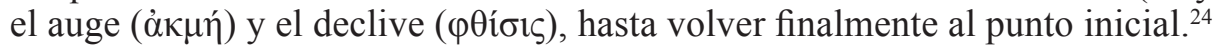

El deslizamiento de una a otra forma de politeia $(\mu \varepsilon \tau \alpha \beta$ o $\lambda \tilde{\eta})$ era inevitable a causa de la debilidad inherente en cada forma simple de gobierno, que queda enunciada en función del número de gobernantes: la monarquía (el gobierno de uno), la aristocracia (el gobierno de unos pocos) y la democracia (el gobierno de muchos). ${ }^{25}$ Aunque estas tres formas puras de gobierno servían con corrección al Estado en sus inicios, se veían sometidas a una decadencia interna que las corrompía hasta que el gobierno finalmente se encontraba en manos de la versión pervertida: la tiranía, la oligarquía y la oclocracia, respectivamente. ${ }^{26} \mathrm{El}$ eco de esta formulación polibiana puede detectarse en la reflexión ofrecida por J. Adams cuando afirma que "el rey, la aristocracia y el pueblo, tan pronto como se sienten seguros de poseer su poder, comenzarán a abusar de él". ${ }^{27}$

No cabe duda de que el futuro presidente de EE.UU. fue uno de los grandes defensores del modelo polibiano y, de hecho, en la introducción de su trabajo $A$ Defence of the Constitutions of Government of the United States of America, reconoce su deuda no hacia Montesquieu, sino hacia el historiador griego al declarar: "deseo reunir las opiniones y razonamientos de los filósofos, políticos e historiadores, que han recogido un amplio número de opiniones sobre los hombres y las sociedades. No se puede negar que todos estos personajes están unidos en Polibio". ${ }^{28}$ Ahora bien, su interés por el modelo polibiano, caracterizado por un gran pragmatismo, revela una absoluta falta de preocupación por conocer y recomponer el contexto político en el que se enmarca la obra del historiador griego, una indiferencia habitual entre los estadistas contemporáneos y comprensible si consideramos que su aproximación al mundo antiguo no tuvo una finalidad científica ni persiguió un objetivo histórico, sino que respondía a las necesidades políticas del momento y se conformaba con recuperar o reinterpretar aquellos modelos que podían servir de instrumento legitimador de las propuestas defendidas.

J. Adams considera que Polibio es más caritativo que grandes filósofos como Maquiavelo, Rousseau, Beccaria o Rochefoucault, porque el historiador griego concede un margen de confianza y buen ejercicio de poder a los responsables de la primera fase de todo gobierno simple. ${ }^{29} \mathrm{Y}$ así, los primeros reyes fueron honestos y sabios administradores, la primera aristocracia, según J. Adams, queda descrita por Polibio como moderada, cauta y patriota al expulsar al tirano, forma degenerada del gobierno real, y al pueblo, al menos en la primera generación una vez depuesta la oligarquía, digno de elogio por su decoro. Pero el monarca, la aristocracia y el pueblo usaron de modo abusivo su poder tan pronto como se sintieron seguros de su estatus

24 Plb. 6.9.10, 6.51.4; Cic. Resp. 1.28. Antes que Polibio, Heródoto ya recoge las tres formas simples de gobierno (Hdt. 3.80-82) así como Tucídides (Th. 8.97), Platón (Pl. Lg. 710e, 712c) y Aristóteles (Arist. Pol. 1279a). Véase Walbank 1957, vol. I, 640, 643-648.

25 Plb. 6.3.5, 6.4.6-11.

26 Adams 1787 (Letter XXXI Polybius). Los padres fundadores manifiestan igualmente su deuda con Platón a quien reconocen, antes que a Polibio, como el primer autor que identifica esas tres formas simples de gobierno y su peligrosa evolución degenerada.

27 Adams 1787 (Letter XXXI Polybius).

28 Adams 1787 (Letter XXX Polybius).

29 Adams 1787 (Letter XXXI Polybius). 
y ejercieron el gobierno ya bajo su forma corrupta siguiendo el previsible ciclo vital de la anaciclosis. ${ }^{30}$

Ya en 1763, durante el periodo prerrevolucionario, J. Adams esgrimía el pernicioso efecto de la anaciclosis para mostrar su más absoluta oposición a cualquiera forma de gobierno simple incapaz de proteger a los hombres contra la violencia del poder. ${ }^{31}$ Su postura fue compartida años más tarde por otro padre fundador, J. Wilson, que también desautorizaba este tipo de fórmula política, dibujando el previsible recorrido de la anaciclosis al denunciar la irremediable manifestación de las formas degeneradas de cada gobierno simple: la monarquía derivaría en despotismo, la aristocracia en oligarquía y, en el caso de la democracia, su degeneración en anarquía llevaría a cada individuo a buscar tan solo su propio beneficio arriesgando su vida misma, su propiedad y su reputación. La corrupción radicaba en la propia esencia de estas formas de gobierno pues, según el delegado de Pensilvania, si en una monarquía había fuerza y vigor, había también peligro de que no fuera empleada para obtener la felicidad y la prosperidad del Estado. En una aristocracia se podía esperar la sabiduría derivada de la educación y la experiencia, pero también recelo y disensiones entre los nobles y la opresión de los órdenes más bajos. En una democracia se exaltaba la virtud pública y la pureza aunque, por otro lado, sus representantes se mostraban a menudo negligentes y la ejecución de sus medidas, débil. ${ }^{32}$

La anaciclosis, como fórmula que rige la evolución política, vuelve a estar presente de manera expresa en el capítulo que, en su obra $A$ Defence of the Constitutions, J. Adams dedica al historiador griego por su utilidad en la futura articulación política del estado americano; por ello, incluso parafrasea fragmentos del libro sexto de las Historias. Se puede intuir el peso de la anaciclosis en la reflexión política de J. Adams, si bien no llega a ser explícitamente mencionada, cuando concluye mediante una metáfora:

Toda forma de gobierno que sea simple, pronto degenerará en ese vicio que está aliado a él y naturalmente lo asistirá, sencillamente porque es inestable. Porque al igual que el óxido es la perdición natural del hierro y los gusanos de la madera, en cada forma simple de gobierno hay un cierto vicio implantado por la mano de la naturaleza, y por ella ordenada para acompañarlo. El vicio del gobierno real es la monarquía; el de la aristocracia, la oligarquía; y el de la democracia, la rabia y la violencia; y hacia ese vicio todos ellos necesariamente tienden a degenerar. ${ }^{33}$

Se trata de un símil ya empleado por Polibio cuando enuncia que "cada variedad de constitución simple y basada en un principio único resulta caduca: degenera muy pronto en la forma viciosa inferior que la sigue naturalmente" y propone, a modo de comparación, "el orín, para el hierro, y la carcoma y ciertos gusanos para la madera, que llegan a destruir estos materiales".34

Mediante la observación directa de la Historia Antigua, J. Adams se pertrechaba de ejemplos prácticos de la anaciclosis, aprendía a detectar la fase en la que se

\footnotetext{
Adams 1787 (Letter XXXI Polybius).

Adams 1763. Una reflexión similar aparece en Publius 1788a.

Wilson 1790.

Adams 1787 (Letter XXXI Polybius).

Plb. 6.10 (trad. Balasch, BCG 1981).
} 
encontraba un Estado y a prever el siguiente paso en su evolución, comprobaba la naturaleza inexorable del cambio político, la tendencia a la corrupción de cada tipo de gobierno simple y los errores inherentes a su naturaleza. Esta experiencia heredada permitía evitar esas alternativas fallidas como posibles soluciones de gobierno y defender como principio absolutamente imprescindible en el nuevo Estado la puesta en marcha de un sistema que garantizase el equilibrio entre los tres poderes mediante el sistema de pesos y contrapesos. La composición del ejecutivo fue uno de los asuntos que generó más debate en la convención constituyente ${ }^{35}$ pues el carácter unipersonal que algunos compromisarios, como B. Franklin o J. Wilson, proponían evocaba la monarquía británica de la que habían logrado liberarse tras la guerra de la Independencia. Finalmente, la aprobación de un ejecutivo presidencialista quedó justificada, al menos desde el punto de vista teórico, como un modo de incluir en el nuevo Estado la monarquía entendida como una de las tres formas simples necesarias para asegurar la aplicación de la fórmula mixta, considerada, como ya he señalado, el modelo de gobierno ideal. ${ }^{36}$

T. Jefferson también compartía esa función didáctica de la Historia, útil para prevenir a sus contemporáneos sobre la tiranía, consecuencia del devenir natural de los gobiernos:

Es cierto que ciertas formas de gobierno están mejor calculadas que otras para proteger a los individuos en el libre ejercicio de sus derechos naturales y están, al mismo tiempo, mejor protegidas contra la degeneración. Según muestra la experiencia, incluso las mejores formas de gobierno, las que confían en el poder que tienen, se pervierten en tiranía. El medio más efectivo para prevenir esto sería iluminar, tanto como sea posible, las mentes del pueblo y sobre todo darles a conocer aquellos hechos que exhibe la Historia, la experiencia que poseen otras épocas y naciones. Así serían capaces de reconocer la ambición bajo todas sus formas y estar preparados para ejercer sus poderes naturales y derrotar sus propósitos. $^{37}$

Para obtener un resultado óptimo, recomendaba además la creación de universidades americanas pues, si el estudiante se acercaba a la historia de las repúblicas antiguas en el corrupto entorno de las monarquías europeas, los posibles efectos beneficiosos de esas lecturas sobre el pasado se habrían desperdiciado. ${ }^{38}$

Para los padres fundadores, la tiranía como forma de gobierno no solo era el resultado de la degeneración natural de un gobierno unipersonal, sino de cualquier forma de gobierno simple. Así lo sostiene J. Madison en el número 47 de El Federalista:

... la acumulación de todos los poderes, legislativo, ejecutivo y judicial, en las mismas manos, ya sea de uno, unos pocos o muchos, y ya sea hereditaria, nombrada

\footnotetext{
Protagonizó la sesión del 1 de junio de 1787.

No solo los ya mencionados J. Wilson, A. Hamilton, J. Madison y J. Adams se erigen en firmes defensores del gobierno mixto, sino que merecen ser mencionadas otras figuras menos conocidas, pero igualmente protagonistas del período constituyente, como G. Wythe. J. Dickinson, P. Butler y J. Rutledge. Sus intervenciones a este respecto fueron decisivas durante las sesiones de la convención Constituyente celebradas durante los días 1 y 2 de junio de 1787 .

37 Jefferson 1779.

38 Jefferson 1785.
} 
por uno mismo o electiva, puede ser justamente declarada la definición misma de tiranía. ${ }^{39}$

Esta formulación política, esgrimida una y otra vez en el periodo constituyente para promover las bondades de un gobierno mixto, descansa en el principio de la anaciclosis. J. Adams asumía la rotación de los gobiernos como el orden de la naturaleza mediante el cual cambian, se transforman y regresan al mismo punto inicial $\mathrm{y}$, por lo tanto, se detecta incluso en los gobiernos democráticos idéntico riesgo de caer en la tiranía por el peligro que suponía otorgar el poder legislativo al pueblo, que padecía los mismos vicios, locuras y debilidades de un individuo. ${ }^{40}$ Consideraba, por ejemplo, que la caída de Atenas y otras ciudades se debía a la existencia de gobiernos en manos de la asamblea del demos ${ }^{41}$ y por ello no dudó en describir como modelo absolutamente pernicioso la democracia ateniense de tiempos de Pericles. ${ }^{42}$ Sostenía el estadista americano que las aspiraciones de unos pocos, amparadas y ocultas tras las licencias de muchos, tuvieron como consecuencia -no solo en Atenas, sino en un gran número de póleis griegas- una sucesión de actos bárbaros que culminaron en la Guerra del Peloponeso. ${ }^{43}$ El exceso de democracia ejemplificado en Atenas mostraba el desenlace previsible de la anaciclosis: inestabilidad, violencia, corrupción e injusticia fruto de la intervención exclusiva del demos, hacia el que manifiesta una profunda antipatía:

Un populacho usurpador es un incauto ruin que se vende confiado a cualquier tirano, con un instinto tan ciego como los gusanos que mueren mientras hilan con su capullo el magnífico hábitat de los que son de un orden superior. ${ }^{44}$

Al igual que J. Adams, otros reputados federalistas consideraron la democracia asamblearia como un mero tránsito hacia la tiranía. ${ }^{45}$ A. Hamilton, delegado por Nueva York en la convención constituyente, descartaba cualquier forma de gobierno simple como una opción política viable y advertía: "si nos inclinamos demasiado hacia la democracia, pronto caeremos en la monarquía", ${ }^{46}$ una deducción que solo cobra su pleno sentido teniendo presente el recorrido polibiano de la anaciclosis. En una reflexión similar, el gran clasicista de la convención, J. Madison, alertaba del peligro de corrupción de la república si todo el legislativo se confiaba en las manos de un solo colectivo, ${ }^{47}$ y J. Wilson, delegado por Pensilvania, sostenía que una legislatura con una sola cámara estaba condenada a unir todas las cualidades perniciosas de un mal gobierno: "produce debilidad general, inactividad, y confusión unido a súbitos y violentos ataques de despotismo, injusticia y crueldad". ${ }^{48}$

Como argumento adicional para rechazar la democracia -y en el capítulo que en A Defence of the Constitutions dedica a Polibio-, J. Adams advertía del peligro que suponía conceder todo el poder legislativo a una sola asamblea en la que participaran

\footnotetext{
Publius 1788a.

40 Adams 1776.

41 Adams 1787 (Letter XLI Athens).

42 Adams 1787 (Letter XXIV Dr. Swift).

43 Publius 1787; Adams 1787 (Letter XLI Athens).

44 Adams 1787 (Letter XXIV Swift).

45 Farrand 1961 (June 19, 1787).

46 Farrand 1961 (June 26, 1787).

47 Publius 1788a.

$48 \quad$ Wilson 1790.
} 
sin distinción individuos de la elite y el pueblo con el fin de destruir toda igualdad y libertad, incluso con el consentimiento y aclamación del propio pueblo. ${ }^{49}$

El único remedio era "arrojar a los ricos y orgullosos a una asamblea separada y atar allí sus manos". ${ }^{50}$ Así quedó legitimado un legislativo bicameral con el establecimiento de una cámara senatorial, órgano que defendería al pueblo de sus propios errores, sobre todo en aquellos momentos en los que, inducido -en palabras de J. Madison- por alguna pasión irregular o con la esperanza de obtener alguna ventaja ilícita, o incluso engañado por individuos - los más pudientes-con intereses propios, podía solicitar medidas que el mismo pueblo terminaría por lamentar e incluso condenar. ${ }^{51} \mathrm{~J}$. Adams también comparte las ventajas derivadas de separar a los poderosos en una cámara específica:

Tendrán mucho más poder, mezclados con los representantes, que separados de ellos. Pero al colocarlos solos, la sociedad aprovecha todas sus capacidades y virtudes; se convierten en un férreo control de esos mismos representantes así como del poder ejecutivo, y quedan incapacitados para hacer daño alguno. ${ }^{52}$

Todas estas prevenciones revelan cuán presente está en las propuestas políticas de los estadistas americanos la amenaza latente de la anaciclosis. Defienden los padres de la nación que el nuevo gobierno debería quedar articulado de tal modo que ninguno de sus órganos prevaleciera sobre los restantes con el fin de preservar las libertades individuales y, sobre todo, evitar la tiranía, ese destino inexorable al que conducía cualquier forma de gobierno simple en el cíclico recorrido de la anaciclosis. El mecanismo más eficaz para evitar este riesgo pasaba por el establecimiento del principio de separación de poderes y el equilibrio de poderes entre el gobierno central y los distintos estados. ${ }^{53} \mathrm{~J}$. Adams defendía que solo el cuidado y la decisión mostrados al mismo tiempo por los gobiernos de los distintos estados federales y el central en la defensa de este equilibrio, ahuyentarían los peligros de la tiranía. ${ }^{54}$ Asimismo, consideraba imprescindible, para asegurar el éxito de la propuesta, reforzar el sistema con un conjunto de pesos y contrapesos como garantes de la independencia, la libertad y la aplicación justa de las leyes. Según el padre fundador, la Historia enseñaba que, si no se establecía el equilibrio de poderes, la ineludible anaciclosis condenaría a la nueva nación a padecer constantes revoluciones:

Si el poder ejecutivo o una parte considerable de él, se deja en manos de una asamblea aristocrática o democrática corromperá la legislatura como el óxido corroe el hierro o el arsénico el cuerpo humano, y cuando se corrompe la legislatura, el pueblo camina hacia la perdición. ${ }^{55}$

\footnotetext{
Adams 1787 (Letter XXXI Polybius).

Adams 1787 (Letter XXXI Polybius).

Publius 1788c. Una opinión similar mantiene Hamilton en la convención ratificadora de Nueva York: Elliot 1968 (June 24, 1788); Adams 1787 (Letter XXIV Swift).

52 Adams 1787 (Letter XXIV Swift).

53 Farrand 1961 (June 28, 1787).

54 Adams 1787 (Letter V Conclusion).

55 Adams 1787, praef. Con toda seguridad sigue aquí a Polibio (Plb. 6.10).
} 
Según J. Adams, había que buscar y preservar esta armonía a pesar de las dificultades, pues se corría el riesgo de perder la libertad para siempre. Pero no todos los padres fundadores participaron del mismo temor a la degeneración política: los antifederalistas defendían, como el mejor antídoto, una sociedad virtuosa gracias a una educación adecuada, acompañada de un ordenamiento jurídico surgido de la voluntad de la mayoría. ${ }^{56}$

\section{Polibio en los círculos intelectuales de las colonias}

La influencia de Polibio, por lo tanto, resulta innegable y tan solo cabe indagar cómo los compromisarios y estadistas de los primeros tiempos de la república accedieron a un conocimiento tan detallado de su obra, que no parece podamos atribuir únicamente a la información proporcionada por Montesquieu. Dada la importancia de las disciplinas clásicas en los estudios universitarios más solicitados entonces, Derecho y Teología, no resulta sorprendente que los colonos de cierto nivel educativo, y más aún los políticos más activos y filoclásicos, accedieran directamente al texto original en griego, como sin duda hicieron J. Madison, J. Wilson, A. Hamilton, E. J. Randolph o J. Dickinson. ${ }^{57}$ Así lo aconsejaba T. Jefferson, por ejemplo, pues de este modo podría obtenerse un mayor beneficio del legado clásico. ${ }^{58}$ De hecho, en la publicación de las actas de la convención constituyente y en las celebradas en cada uno de los estados para aprobar la Constitución, los textos griegos y latinos se dejaban sin traducir, pues se presuponía en los lectores suficiente formación clásica.

T. Jefferson incluso disponía de numerosas ediciones de Polibio en su biblioteca particular y sabemos por su correspondencia que durante su estancia en Europa fue comprando ejemplares de sus Historias destinadas no solo al consumo propio, sino que también las envió a sus amigos presentes en la convención. Solicitó una edición italiana a F. Mazzei en febrero de $1787 .{ }^{59}$ En agosto había enviado una edición a P. Carr y un mes más tarde escribió a G. Wythe para que le procurase una copia en la mejor edición que encontrara. ${ }^{60}$ En marzo del año siguiente, solicitó a Van Damme por carta una edición holandesa de $1548^{61}$ y un año más tarde otra publicada en 1584 . Dos meses más tarde escribió a J. Trumbul solicitando copias del Polibio de J. Hampton. $^{62} \mathrm{La}$ avidez de T. Jefferson responde a su extrema preocupación por

56 Como G. Mason en la convención constituyente: Rutland 1970, vol. III, June 4, 1787; Farrand 1961, May 31, 1787; Farrand 1961, June 25, 1787 (intervención del antifederalista C. Pinckney). Posturas similares en la convención de Virginia: Elliot 1968, III (June 5, 1788, P. Henry; June 10, 1788, J. Monroe); en la de Carolina del Sur: Elliot, 1968, IV (May 14, 1788, C. Pinckney). En la prensa, los antifederalistas mostraron la misma oposición: Centinel 1787; Cato 1787; A Federal Farmer 1788, Philadelphiensis 1787; Helvidius Priscus 1787; Sidney 1788; Gerry 1788. La réplica de estos intelectuales a ese temor federalista no buscó legitimación en el mundo clásico, si bien los antifederalistas recurrieron a la recuperación del mundo antiguo para defender la autonomía de los estados como un modelo político factible con garantías de éxito, tal y como mostraba, de manera particular, el triunfo griego en las Guerras Médicas.

57 Hiden 1941, 26-28; Middlekauff 1961, 65; Wiesen 1977, 63.

58 Jefferson 1779.

59 Jefferson $1787 \mathrm{a}$.

60 Jefferson $1787 \mathrm{~b}$.

61 Jefferson $1788 \mathrm{a}$

62 Jefferson 1788 b. La traducción inglesa de J. Hampton fue publicada con el título The General History of Polybius, London, 1756-61. 
acceder a un texto lo más fiable posible, pues constituiría el aval de sus propuestas políticas, en un momento en el que la filología clásica como ciencia comenzaba a dar sus primeros pasos y las ediciones presentaban no pocos problemas. Era preciso realizar lecturas cruzadas con el fin de cotejar los pasajes más conflictivos. ${ }^{63}$

J. Adams también fue un lector empedernido de Polibio y en su biblioteca disponía de algunas ediciones de su obra. ${ }^{64}$ Durante las sesiones de la convención constituyente, sus escritos, sembrados de constantes referencias a la teoría polibiana de la anaciclosis, como hemos visto, circularon entre los compromisarios reunidos en Filadelfia. De hecho, la sección dedicada a los antiguos gobiernos dentro de la ya mencionada The Defense of the Constitutions fue publicada en enero de 1787 y ya había llegado a Estados Unidos en marzo, justo antes de la apertura de la convención constituyente para ser reimpresa inmediatamente en Boston, Nueva York y Filadelfia, de modo que cualquier delegado de la convención que hubiera leído este trabajo de J. Adams había podido acceder siquiera de manera indirecta a Polibio. ${ }^{65}$ De todos modos, todo aquel que no leyó a Polibio en griego disponía de traducciones en francés, italiano, latín e inglés, de consulta mucho más cómoda.

\section{Esparta y Roma, inspiración del futuro gobierno mixto}

Así fue como Polibio ofreció directamente a los padres fundadores no solo ejemplos de los peligros derivados de la anaciclosis, sino también una alternativa eficaz de gobierno que vino de la mano de dos estados del mundo antiguo presentados de manera idealizada por el historiador griego y convertidos por los padres fundadores, a partir de la imagen polibiana, en paradigmas clásicos del gobierno mixto: la Esparta de Licurgo y la Roma republicana. Y así, según detalla J. Adams en el capítulo dedicado a Lacedemonia, Licurgo instituyó en Esparta un gobierno mixto tras la observación de las leyes de la naturaleza que dictan la inestabilidad de cualquier gobierno simple y su degeneración hacia su versión corrompida. ${ }^{6}$ Para evitar la anaciclosis, Licurgo unió lo mejor que ofrecía cada uno de los gobiernos simples en su fase inicial, dispuestos con un equilibrio tal que ninguno de ellos pudiera superar el resto. Según Adams, fue el establecimiento de este sistema de pesos y contrapesos el que permitió a los lacedemonios disfrutar de una libertad inigualable. ${ }^{67}$

La república romana también fue recuperada como arquetipo, pues había logrado salvar el inevitable ciclo de la anaciclosis al instituir una constitución mixta en el que la monarquía quedaba representada bajo la forma de un ejecutivo electivo en la persona de los cónsules, del mismo modo que la aristocracia en el Senado y la democracia en las distintas asambleas populares. Parafraseando a Polibio, ${ }^{68}$ el equilibrio era tal y la república estaba administrada con tanta igualdad por parte de

63 Por ejemplo, J. Adams (1814) describe una labor de auténtica crítica textual ante la falta de ediciones fiables: "some thirty years ago I took upon me the severe task of going through all (scl. Plato's) works. With the help of two Latin translations and one English and one French translation, and comparing of the most remarkable passages with the Greek, I labored through the tedious toil".

64 Incluso su mujer Abigail incluye una cita de Polibio en una carta dirigida a su marido: Adams 1774.

65 Martínez Maza 2013, 31-32.

66 Adams 1787 (Letter XL Lacedaemon).

67 Adams 1787 (Letter XXXI Polybius).

68 Plb. 6.11. 
esos tres poderes que no era posible, incluso para un ciudadano romano, confirmar si el gobierno era aristocrático, democrático o monárquico:

... porque, si nos fijamos en el poder de los cónsules, el gobierno parece exclusivamente monárquico; si lo hacemos en el del senado, aristocrático; y cuando cualquiera hacia lo mismo con las asambleas populares, podría identificarlo con un gobierno indudablemente democrático. ${ }^{69}$

Para explicar esta imagen que el espectador obtiene al contemplar la república romana, J. Adams incluye una minuciosa descripción de las competencias sustanciales atribuidas a cada uno de los órganos que integran el estado romano. Dos elementos concitan la atención del estadista en el ordenamiento político descrito por Polibio: por un lado, las atribuciones concedidas a cada una de las ramas "para ayudarse y frenarse mutuamente" y, por otro, la aplicación de un sistema de control y equilibrio de poderes que considera un antecedente clásico e inspiración del mecanismo que se pretende establecer en la nueva república de los EE. UU. De este modo, según J. Adams:

Cuando alguna de sus ramas se vuelve ambiciosa y, sobrepasando sus límites, pretende un poder injustificable, como está sujeta al control de las otras dos, no puede hacer gala de ningún exceso de poder o arrogancia, sino que las tres deben permanecer dentro de los límites señalados por la constitución. ${ }^{70}$

Esta visión de ecos polibianos, que presenta a la república romana como el sistema de gobierno más perfecto, fue aceptada de manera unánime por los estadistas americanos, aunque con las necesarias matizaciones relativas, sobre todo, a la separación de poderes y al funcionamiento eficaz del sistema de controles y equilibrios. En palabras de J. Adams, si algún síntoma de debilidad mostraba el arquetipo romano era la ausencia de una clara distribución del poder. ${ }^{71}$ El ejecutivo nunca estuvo lo suficientemente separado del legislativo, ni existía un control mutuo definido con suficiente precisión. Para J. Adams, aun siendo el poder legislativo natural y necesariamente soberano y supremo, el ejecutivo debía disponer de capacidad y fortaleza suficientes para defenderse de las intervenciones de aquel, pues solo así podrían garantizarse los derechos y libertades de la población. Criticaba el padre fundador la ausencia de derecho de veto, por ejemplo, que permitiera a los cónsules intervenir y resolver las desavenencias entre el pueblo y el Senado. Esta prerrogativa suponía un instrumento esencial para garantizar el equilibrio de poderes y evitar la degeneración del sistema de gobierno. Fue la ausencia de este elemento el que explicaría, asimismo, la caótica situación política que percibe en el gobierno democrático ateniense de tiempos de Pericles. ${ }^{72}$

En definitiva, el análisis de las distintas constituciones mixtas del mundo clásico permitió a los padres fundadores proponer una estructura política libre de las lacras observadas en el pasado, de la degeneración connatural de los gobiernos gracias a una clara separación de los poderes ejecutivo, legislativo y judicial. La constitución

\footnotetext{
Adams 1787 (Letter XXX Polybius).

Adams 1787 (Letter XXX Polybius).

Adams 1787 (Letter XXX Polybius).

Adams 1787, praef. (Letter XLI Athens).
} 
federal y las de los estados que conformaban la nueva república serían, en consecuencia, versiones mejoradas y sistemas superiores gracias a la experiencia ofrecida por la Historia. ${ }^{73}$

\section{Referencias bibliográficas}

Adams, J.

(1774): “[Letter] To John Adams", [en] C. C. Little - J. Brown (eds.), Letters of Mrs. Adams, the Wife of John Adams, Boston, 1840, vol. I, 21.

(1763): “An Essay on Man's Lust for Power”, August 29, [en] R. J. Taylor (ed.), The Papers of John Adams, Cambridge (MA), 1977, vol. I, 81-84.

(1776): Thoughts on Government Applicable to the Present State of the American Colonies, [en] Ch. F. Adams (ed.), The Works of John Adams, Second President of the United States: with a Life of the Author, Notes and Illustrations, Boston, 1850-1856, vol. IV, 65-93.

(1787): A Defence of the Constitutions of Government of the United States of America, New York.

(1814): “[Letter] To Thomas Jefferson", July 16, 1814, [en] L. J. Cappon (ed.), The Adams-Jefferson Letters: The Complete Correspondence between Thomas Jefferson and Abigail and John Adams, Chapel Hill, 1959, 434-439.

Beal Davis, R. (1978): Intellectual Life in the Colonial South 1585-1763, Knoxville, 3 vols.

Boyd, J. P. (ed.), (1950-): The Papers of Thomas Jefferson, Princeton, vols. 1-20.

Butterfield, L. H. (ed.), (1961): The Diary and Autobiography of John Adams, Cambridge (MA), 4 vols.

Carrese, P. O. (2003): The Cloaking of Power: Montesquieu, Blackstone and the Rise of Judicial Activism, Chicago (https://doi.org/10.7208/chicago/9780226094830.001.0001).

Cato (1787): "Letter V", The New York Journal, November 22, [en] Ketcham (ed.), 2003, 317-321.

Centinel (1787): "Letter I (Philadelphia Independent Gazetteer, October 5", [en] Ketcham (ed.), 2003, 227-237.

Cohler, A. M. (1988): Montesquieu's Comparative Politics and the Spirit of American Constitutionalism, Lawrence.

Cullen, C. T. (ed.), (1962): The Papers of Thomas Jefferson, Princeton.

Elliot, J. (ed.), (1836-45): The Debates in the Several State Conventions on the Adoption of the Federal Constitution, New York.

Farrand, M. (ed.), (1966): The Records of the Federal Convention of 1787, New Haven, 4 vols.

A Federal Farmer (1787): “Essays by a Federal Farmer (Maryland Gazette, October 9”, [en] Storing (ed.), 1981, vol. 5, 5-60.

Gerry, E. (1788): "On the New Constitution, and on the Federal and State Conventions by a Columbian Patriot", [en] P. Leicester Ford (ed.), Pamphlets on the Constitution of the United States, published during its discussion by the People 1787-1788, 1998, New York, 2-23.

Gummere, R.

(1957): “The Classical Politics of John Adams", Boston Public Library Quarterly 9, 167182.

73 Adams 1787 (Letter XXX Polybius). 
(1960): "Some Classical Side Lights on Colonial Education", The Classical Journal 55, 223-232.

Harrington, J. (1656): The Commonwealth of Oceana and a System of Politics, London (=ed. J. G. A. Pocock, Cambridge, 1992), (https://doi.org/10.1017/CBO9781139137126).

Helvidius Priscus (1787): "Sin título", Independent Chronicle, December 27, [en] Storing (ed.), 1981, vol. IV, 152.

Hiden, M. W. (1941): "Education and the Classics in the life of Colonial Virginia", Virginia Magazine of History and Biography 49, 21-28.

Jefferson, T.

(1779): “A Bill for the More General Diffusion of Knowledge”, June 18, [en] Boyd (ed.), 1950, vol. 2, no 79, 526-535.

(1785): “[Letter] To John Banister Jr.”, October 15, [en] Boyd (ed.), 1953, vol. 8, 635637.

(1787a): “[Letter] To Philip Mazzei”, February 16, [en] Boyd (ed.), 1955, vol. 11, 159.

(1787b): “[Letter] To George Wythe”, September 16, [en] Boyd (ed.), 1955, vol. 12, 127 129.

(1788a): “[Letter] To Van Damme”, March 23, [en] Boyd (ed.), 1955, vol. 12, 688.

(1788b): "[Letter] To John Trumbull”, May 18, [en] Boyd (ed.), 1956, vol. 13, 178-180.

Ketcham, R. (ed.), (2003): The Anti-Federalist Papers and the Constitutional Convention Debates. The Clashes and the Compromises that Gave Birth to Our Form of Government, Toronto.

Lutz, D. (1988): The Origins of American Constitutionalism, Baton Rouge.

Madison, J. (1783): "Report on Books for Congress", January 23, [en] R. A. Rutland et al. (eds.), The Papers of James Madison, Chicago, 1976-1977, vol. VI, 62-115.

Martínez Maza, C. (2013): El espejo griego: Atenas, Esparta y las ligas griegas en la América del periodo constituyente (1776-1787), Barcelona.

McLean, J. - McLean, A. (eds.), (1788): The Federalist: a Collection of Essays, Written in Favour of the New Constitution, as Agrees upon by the Federal Convention, September 17, 1787, in two volumes, New York.

Middlekauff, R. (1961): "A Persistent Tradition: The Classical Curriculum in EighteenthCentury New England”, William and Mary Quarterly 18, 53-66 (https://doi. org/10.2307/1922807).

Montesquieu, Barón de (1748): De l'esprit des lois, Genève, 2 vols.

Nelson, E. (2004): The Greek Tradition in Republican Thought (=Ideas in Context 69), Cambridge (https://doi.org/10.1017/CBO9780511490644).

Philadelphiensis (1787): "Letter V", Philadelphia Independent Gazetteer, December 19, [en] Storing (ed.), 1981, vol. IV, 152.

Publius

(1787): "Concerning Dangers from Dissensions between the States (The Federalist Papers 6, November 14)", [en] McLean - McLean (eds.), 1788, vol. I, 25-32.

(1788a): "The Particular Structure of the New Government and the Distribution of Power Among Its Different Parts (The Federalist Papers 47, January 30)", [en] McLean McLean (eds.), 1788, vol. II, 92-101.

(1788b): "The Structure of the Government Must Furnish the Proper Checks and Balances Between the Different Departments (The Federalist Papers 51, February 6)", [en] McLean - McLean (eds.), 1788, vol. II, 116-122.

(1788c): "The Senate Continued (The Federalist Papers 63, March 1)", [en] McLean McLean (eds.), 1788, vol. II, 191-201. 
Rahe, P. A. (1992): Republics Ancient and Modern: Classical Republicanism and the American Revolution, Chapel Hill.

Rutland, R. A. (ed.), (1970): The Papers of George Mason, Chapel Hill.

Sidney (1788): "Sin título", New York Patriotic Daily, June 13-14, [en] Storing (ed.), 1981, vol. VI, 103.

Storing, J. (ed.), (1981): The Complete Anti-Federalist, Chicago, 6 vols.

Swift, J. (1701): Discourse of the Contests and Dissensions between the Nobles and Commons of Athens and Rome, London (printed for John Nutt).

Vile, M. J. C. (1998): Constitutionalism and the Separation of Powers, Indianapolis.

Walbank, F. W. (1957): A Historical Commentary on Polybius, Oxford.

Wiesen, D. S. (1977): "Ancient History and Early American Education", [en] S. Ford Wiltshire - L. B. Wright (eds.), The Usefulness of Classical Learning in the EighteenthCentury. Papers presented at the 107 $7^{\text {th }}$ annual Meeting of the American Philological Association 1975, State College, 1977, 53-69.

Wilson, J. (1790): "Lectures on Law (part. 1). Of Government”, [en] K. L. Hall - M. D. Hall (eds.), Collected Works of James Wilson, Indianapolis, 2007, vol. I, 689-717. 
